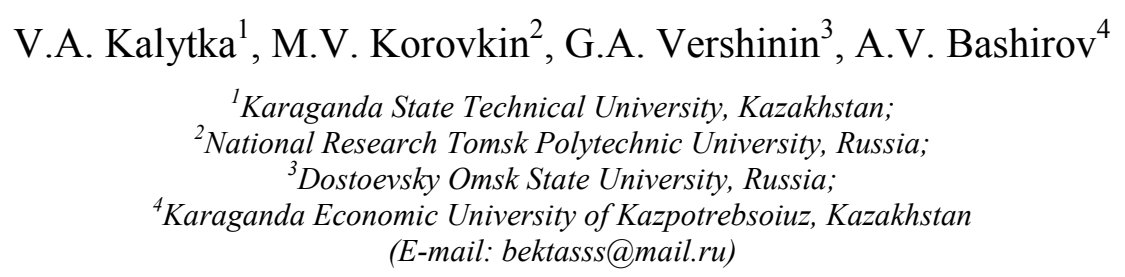

\title{
Mechanism of non-linear space-charge polarization in solid dielectrics
}

\begin{abstract}
The nonlinear kinetic phenomena during shaping of volume-charge polarization in hydrogen bonded crystals (HBC) is investigated by the methods of quasi - classical kinetic theory. From the solution of the nonlinear system of equations, Fokker - Planck and Poisson, in case of blocking electrodes, it is established that the mathematical description of the relaxation of polarization in $\mathrm{HBC}$, in the region of weak fields $(100-1000 \mathrm{kV} / \mathrm{m})$ and high temperatures $(\mathrm{T}>350 \mathrm{~K})$ linear approximation of the perturbation theory is sufficient. The coefficients of diffusion and mobility are calculated with respecting of both transitions mechanisms (thermally activated and tunneling) of the protons through potential barrier of parabolic shape. Proton - proton and proton - phonon interaction is not considered. The proposed scheme for solving the kinetic equation can be applied to other, similar to the HBC type and properties of the crystal lattice crystals with ionic conductivity.
\end{abstract}

Keywords: proton semiconductors and dielectrics; hydrogen bonded crystals (HBC); proton relaxation and conductivity; non-linear system of equations of Fokker - Planck and Poisson; relaxation modes; polarization frequency harmonics.

\section{Introduction}

Hydrogen bonded crystals (HBC) by electrophysical properties in the range of temperature $T=50-450 \mathrm{~K}$, at intensities polarizing field $E_{0} \approx 10^{5} \div 10^{6} \frac{\mathrm{V}}{\mathrm{M}}$, are classified as proton semiconductors and dielectrics exhibited the property of proton conductivity caused by diffusion transfer of hydrogen ions (protons) along hydrogen links towards the electric field lines [1].

For electrical industry, insulation and cable technology the certain scientific interest is in the study of the kinetics of space - charge polarization in HBC in the range of high temperature $(T=100-450 \mathrm{~K})$ when the mechanism of dielectric relaxation in crystal is determined by thermally activated, and at low temperatures $(T=50-100 \mathrm{~K})$, by tunneling junctions of protons [2]. This polarization type is well studied experimentally, but its theoretical description remains unsatisfactory $[2,3]$.

Currently, two models - microscopic and phenomenological - were applied during the investigating of processing of space charge accumulation. The first model is based on the concept of charge transfer in a multi-well potential relief and to define the space - charge distribution is necessary, in general, to solve the non-linear system of $\left(\approx 10^{7}\right)$ equations, which is not analytically possible [2]. The solution obtained by such a system only for homogeneous low electric fields [1]. The second model is upon the description of diffusion transfer charge in the substance and it is built on the solving of the system of equations of Fokker - Planck and Poisson [2]. Since the initial phenomenological model equations are nonlinear by polarizing field, analytical solutions alone were gotten under the condition of their linearization [3].

\section{The nonlinear properties of kinetic equation of proton relaxation}

An analytical investigation of the influence of kinetic equation's nonlinearities [2], and establishment of the space charge relaxation mechanism on that basis will be built on the expansion of the space charge density and the dimensionless electric field $z(\xi ; \tau)=\frac{E(x ; t)}{E_{0}}$ in the non-dimensional power series $[2,3]$

$$
\rho(\xi ; \tau)=\sum_{\mathrm{k}=1}^{+\infty} \gamma^{\mathrm{k}} \rho_{\mathrm{k}}(\xi ; \tau), z(\xi ; \tau)=\sum_{\mathrm{k}=0}^{+\infty} \gamma^{\mathrm{k}} \mathrm{z}_{\mathrm{k}}(\xi ; \tau)
$$

Turning in (1) to an expansion in the frequency harmonics $\rho^{(r \omega)}(\xi ; \tau)$ we have 


$$
\begin{gathered}
\rho(\xi ; \tau)=\sum_{r=1}^{+\infty} \sum_{\mathrm{k}=r}^{\infty} \gamma^{\mathrm{k}} \rho_{\mathrm{k}}^{(r \omega)}(\xi ; \tau), \rho(\xi ; \tau)=\sum_{r=1}^{+\infty} \rho^{(r \omega)}(\xi ; \tau), \\
\rho_{\mathrm{k}}^{(r \omega)}(\xi ; \tau)=\sum_{n=1}^{+\infty} \mathfrak{R}_{k}^{(r \omega)}(n, \tau) \cos \left(\frac{\pi \mathrm{n} a}{\mathrm{~d}} \xi\right) \\
\rho^{(r \omega)}(\xi ; \tau)=\sum_{\mathrm{k}=r}^{\infty} \sum_{\mathrm{n}=1}^{+\infty} \gamma^{\mathrm{k}} \mathfrak{R}_{\mathrm{k}}^{(r \omega)}(\mathrm{n}, \tau) \cos \left(\frac{\pi \mathrm{n} a}{\mathrm{~d}} \xi\right) .
\end{gathered}
$$

Function $\rho_{k}^{(r \omega)}(\xi ; \tau)$ is multiple to frequency $(r \omega)$ relaxation mode $k$-th order perturbation theory and $\mathfrak{R}_{\mathrm{k}}^{(r \omega)}(n, \tau)$ - the complex amplitude of the relaxation mode $n$-th order (the number $n$ of relaxation waves) $\rho_{n, k}^{(r \omega)}(\xi ; \tau)=\mathfrak{R}_{k}^{(r \omega)}(\mathrm{n}, \tau) \cos \left(\frac{\pi \mathrm{n} a}{\mathrm{~d}} \xi\right)$. In (1.1)-(1.3) is $\xi=\frac{x}{a}$ - a dimensionless coordinate; $a$ - lattice constant; $d$ - crystal thickness. Dimensionless time $\tau=W^{(0)} t$ is expressed by the zero field component of proton velocity transition probability through the potential barrier [4]

$$
W^{(0)}(T)=\frac{v_{0}}{2}\left(\exp (-X)+\left\langle D^{(0)}\right\rangle\right),\left\langle D^{(0)}\right\rangle=X \frac{\exp (-\Lambda)-\exp (-X)}{X-\Lambda} .
$$

In (2) $\left\langle D^{(0)}\right\rangle$ - averaged over the energy levels of the quasi-continuous spectrum the parabolic potential barrier transparency; $X=\frac{U_{0}}{k_{B} T}, \Lambda=\frac{\pi \delta_{0} \sqrt{m U_{0}}}{\hbar \sqrt{2}}, m$ - proton mass; $U_{0}$ - activation energy (the high of potential barrier); $v_{0}$ - frequency of natural oscillations of the proton in the unperturbed potential well; $\delta_{0}$ - width of the potential barrier [4]. Small parameter of perturbation $\gamma=\frac{\mu_{m o b}^{(1)} \cdot a E_{0}}{D_{d i f f}^{(0)}}$ [3], according to expressions for the diffusion $D_{d i f f}^{(0)}=a^{2} W^{(0)}$ and the mobility $\mu_{m o b}^{(1)}=\frac{q a^{2} W^{(1)}}{k_{B} T}$ coefficients, where $q-$ charge of a proton [4], is expressed in terms of another small parameter $\varsigma_{0}=\frac{q E_{0} a}{k_{B} T}<<1$ and, on condition $\frac{W^{(1)}}{W^{(0)}}<1$, provided the form $\gamma=\frac{\varsigma_{0} W^{(1)}}{W^{(0)}}$. Here [4]

$$
W^{(1)}(T)=\frac{v_{0}}{2}\left(\exp (-X)+\left\langle D^{(1)}\right\rangle\right),\left\langle D^{(1)}\right\rangle=\frac{\Lambda \exp (-\Lambda)-\mathrm{X} \exp (-X)}{\mathrm{X}-\Lambda} .
$$

The nonlinear system of equations of the phenomenological model has the form $[2,3]$

$$
\begin{gathered}
\frac{\partial \rho}{\partial \tau}=\frac{\partial^{2} \rho}{\partial \xi^{2}}-\theta \rho-\gamma \frac{\partial}{\partial \xi}(\rho z), \frac{\partial \mathrm{z}}{\partial \xi}=\varphi \rho, \\
\rho(\xi, 0)=0,\left.\frac{\partial \rho}{\partial \xi}\right|_{\xi=\left\{0 ; \frac{d}{a}\right\}}=\left.\gamma\left(n_{0}+\rho\right) z\right|_{\xi=\left\{0 ; \frac{d}{a}\right\}}, \int_{0}^{d / a} z(\xi ; \tau) \mathrm{d} \xi=\frac{d}{a} \exp \left(\frac{i \omega}{W^{(0)}} \tau\right) .
\end{gathered}
$$

In (3), (4) the accepted designation $\theta=\varphi \gamma n_{0}, \varphi=\frac{a q}{\varepsilon_{0} \varepsilon_{\infty} E_{0}}, \varepsilon_{\infty}$ - the high frequency dielectric permittivity of the crystal, $n_{0}$ - the equilibrium (balanced) concentration (density) of protons. In the zeroth approximation, in the expressions (1) $z_{0}(\tau)=\exp \left(\frac{i \omega}{W^{(0)}} \tau\right), \rho_{0}(\xi ; \tau)=0$ are accepted [3]. 
We expanded $\rho_{\mathrm{k}}(\xi ; \tau)$ in a Fourier series of orthogonal functions $\cos \left(\frac{\pi \mathrm{n} a}{d} \xi\right)$, on a segment $0 \leq \xi \leq \frac{d}{a}$, with an image $\mathfrak{R}_{k}(n, \tau)=\frac{2 a}{d} \int_{0}^{d / a} \rho_{k}(\xi ; \tau) \cos \left(\frac{\pi \mathrm{n} a}{d} \xi\right) d \xi$, after transformation equations (3), (4), using (1.1), (1.2) and (1.3), we obtain the equation

$$
\begin{gathered}
\frac{\partial \Re_{k}(n, \tau)}{\partial \tau}+\frac{1}{\tau_{n}} \Re_{k}(n, \tau)=\frac{2 a}{d}\left\{n_{0}\left(\left.z_{k-1}\right|_{\xi=\frac{d}{a}} \cdot(-1)^{n}-\left.z_{k-1}\right|_{\xi=0}\right)-\right. \\
\left.-\frac{\pi \mathrm{n} a}{d} \int_{0}^{d / a}\left(z_{0} \rho_{k-1}+\sum_{m=1}^{k-2} z_{m} \rho_{k-m-1}\right) \sin \left(\frac{\pi n a}{d} \xi\right) d \xi\right\},
\end{gathered}
$$

where $\frac{1}{\tau_{n}}=\frac{\pi^{2} n^{2} a^{2}}{d^{2}}+\theta$. The integral of equation (5), taking into account, is equal to

$$
\begin{gathered}
\Re_{k}(n ; \tau)=\left\{-\frac{2 a n_{0}}{d}\left(1-(-1)^{n}\right) \cdot \int_{0}^{\tau} z_{k-1}\left(0 ; \tau^{\prime}\right) \cdot \exp \left(\frac{\tau^{\prime}}{\tau_{n}}\right) d \tau^{\prime}-\frac{2 a}{d} \cdot \frac{\pi \mathrm{n} a}{d} \int_{0}^{\tau}\left(\int_{0}^{d / a}\left(z_{0} \rho_{k-1}+\sum_{m=1}^{k-2} z_{m} \rho_{k-m-1}\right) \times\right.\right. \\
\left.\left.\times \sin \left(\frac{\pi \mathrm{n} a}{d} \xi\right) d \xi \times \exp \left(\frac{\tau^{\prime}}{\tau_{n}}\right)\right) \mathrm{d} \tau^{\prime}\right\} \times \exp \left(-\frac{\tau}{\tau_{n}}\right) .
\end{gathered}
$$

Further conversion function (6) lead to recurrent expression

$$
\begin{gathered}
\Re_{k}(n, \tau)=\frac{8 n_{0} \varphi}{\pi^{2}} \sum_{s=1}^{\infty}\left\{\frac{\sin ^{2}\left(\frac{\pi s}{2}\right) \sin ^{2}\left(\frac{\pi n}{2}\right)}{s^{2}} \times\left[\exp \left(-\frac{\tau}{\tau_{n}}\right) \cdot \int_{0}^{\tau} \Re_{k-1}\left(s, \tau^{\prime}\right) \exp \left(\frac{\tau^{\prime}}{\tau_{n}}\right) d \tau^{\prime}\right]\right\}- \\
-\frac{4 a}{d} \cdot \sum_{s=1}^{\infty}\left\{\frac{n^{2} \cos ^{2}\left(\frac{\pi n}{2}\right) \sin ^{2}\left(\frac{\pi \mathrm{s}}{2}\right)}{n^{2}-s^{2}} \times\left[\exp \left(-\frac{\tau}{\tau_{n}}\right) \cdot \int_{0}^{\tau} \Re_{k-1}\left(s, \tau^{\prime}\right) \exp \left(\left(i \frac{\omega}{W^{(0)}}+\frac{1}{\tau_{n}}\right) \tau^{\prime}\right) d \tau^{\prime}\right]\right\}+ \\
+\frac{8 \varphi}{\pi^{2}} \sum_{m=1}^{k-2} \sum_{p=1}^{\infty} \sum_{l=1}^{\infty}\left\{\frac{n^{2} \cos ^{2}\left(\frac{\pi n}{2}\right) \sin ^{2}\left(\frac{\pi p}{2}\right) \sin ^{2}\left(\frac{\pi l}{2}\right)}{l^{2}\left(n^{2}-p^{2}\right)} \cdot \int_{0}^{\tau} \Re_{k-m-1}\left(p, \tau^{\prime}\right) \Re_{m}\left(l, \tau^{\prime}\right) \exp \left(\frac{\tau^{\prime}}{\tau_{n}}\right) d \tau^{\prime}\right\} \times \\
\quad \times \exp \left(-\frac{\tau}{\tau_{n}}\right) .
\end{gathered}
$$

The first term of the integrand in recurrence formula (7) describes the interactions between the relaxation modes different number $\mathrm{n}$ of the same order $\mathrm{k}$ and leads only to increased amplitudes of the modes in the odd frequency harmonics. The second and third terms of this expression describes the effect of the interaction of different modes and the number $\mathrm{n}$ and order $k$, and lead to the excitation $(k-1) \omega, k \omega-$ harmonics appropriately. According to (7) odd harmonic frequency relaxation modes contain odd numbers $k$, and even harmonics - even $-k$. Thus, the contribution to the dipole moment of the dielectric gives only odd harmonic frequency, etc. The nonlinear interaction relaxation modes have a significant influence on the formation of the space charge at the frequency $\omega$ only at the initial stage when $t \leq \tau_{1}$ relaxation time fashion with $k=1$. Determine the complex amplitudes $\mathfrak{R}_{k}(n, \tau)$ in analytical form at times $t \leq \tau_{1}$ fails, however, when executed in the art $\tau\rangle\rangle \tau_{1}$, provided the relaxation process becomes stationary, periodic and relaxation terms in the expression (7) can be neglected. Since they measure the dielectric losses when losses are connected to the fundamental frequency, it is necessary to determine the complex amplitude $\mathfrak{R}_{k}^{(\omega)}(n, \tau)$ at this 
frequency. Since generation of relaxation modes with complex amplitudes begins with the first-order perturbation theory $[2,3]$

$$
\mathfrak{R}_{1}^{(\omega)}(n, \tau)=-\frac{4 a n_{0}}{d} \times \sin ^{2}\left(\frac{\pi n}{2}\right) \times \frac{\exp \left(i \frac{\omega}{W^{(0)}} \tau\right)}{\frac{1}{\tau_{n}}+i \frac{\omega}{W^{(0)}}},
$$

of the first term of (7) is easily visible

$$
\begin{gathered}
\mathfrak{R}_{2}^{(\omega)}(n, \tau)=-\frac{4 a n_{0}}{d} \times \frac{8 n_{0} \varphi}{\pi^{2}} \times \sin ^{2}\left(\frac{\pi \mathrm{n}}{2}\right) \times \sum_{s=1}^{\infty} \frac{\sin ^{2}\left(\frac{\pi s}{2}\right)}{s^{2}\left(\frac{1}{\tau_{s}}+\mathrm{i} \frac{\omega}{W^{(0)}}\right)} \times \frac{\exp \left(i \frac{\omega}{W^{(0)}} \tau\right)}{\frac{1}{\tau_{n}}+\mathrm{i} \frac{\omega}{W^{(0)}}}, \\
\mathfrak{R}_{3}^{(\omega)}(n, \tau)=-\frac{4 a n_{0}}{d} \times\left(\frac{8 n_{0} \varphi}{\pi^{2}}\right)^{2} \times \sin ^{2}\left(\frac{\pi n}{2}\right) \times\left(\sum_{s=1}^{\infty} \frac{\sin ^{2}\left(\frac{\pi s}{2}\right)}{s^{2}\left(\frac{1}{\tau_{s}}+i \frac{\omega}{W^{(0)}}\right)}\right)^{2} \times \frac{\exp \left(i \frac{\omega}{W^{(0)}} \tau\right)}{\frac{1}{\tau_{n}}+i \frac{\omega}{W^{(0)}}},
\end{gathered}
$$

from which we obtain the recurrence formula

In (9) $\Lambda_{0}=\sum_{s=1}^{\infty} \frac{\sin ^{2}\left(\frac{\pi s}{2}\right)}{\mathrm{s}^{2}\left(\frac{1}{\tau_{s}}+i \frac{\omega}{W^{(0)}}\right)}$

$$
\mathfrak{R}_{k}^{(\omega)}(n, \tau)=-\frac{4 a n_{0}}{d} \times\left(\frac{8 n_{0} \varphi}{\pi^{2}}\right)^{k-1} \times \sin ^{2}\left(\frac{\pi n}{2}\right) \times \Lambda_{0}^{k-1} \times \frac{\exp \left(i \frac{\omega}{W^{(0)}} \tau\right)}{\frac{1}{\tau_{n}}+i \frac{\omega}{W^{(0)}}} .
$$

From (1.3), when $r=1$, we have

$$
\rho^{(\omega)}(\xi ; \tau)=\sum_{k=1}^{\infty} \sum_{n=1}^{+\infty} \gamma^{\mathrm{k}} \mathfrak{R}_{k}^{(\omega)}(n, \tau) \cos \left(\frac{\pi n a}{d} \xi\right) .
$$

Substituting (9) to (10) gives a final

$$
\rho^{(\omega)}(\xi, \tau)=-\frac{4 a n_{0} \gamma}{d\left(1-\frac{8 \varphi n_{0} \Lambda_{0} \gamma}{\pi^{2}}\right)} \times \sum_{n=1}^{+\infty}\left[\frac{\sin ^{2}\left(\frac{\pi n}{2}\right)}{\frac{1}{\tau_{n}}+i \frac{\omega}{W^{(0)}}}\right] \times \cos \left(\frac{\pi \mathrm{n} a}{d} \xi\right) \times \exp \left(\frac{i \omega \tau}{W^{(0)}}\right) .
$$

From (1.2), when $r=1$, when

$$
\rho_{k}^{(\omega)}(\xi ; \tau)=\sum_{n=1}^{+\infty} \mathfrak{R}_{k}^{(\omega)}(n, \tau) \cos \left(\frac{\pi n a}{d} \xi\right),
$$

considering (9), we have

$$
\begin{gathered}
\rho_{k}^{(\omega)}(\xi, \tau)=-\frac{4 a n_{0}}{d} \cdot \frac{8^{k-1} a^{k-1} q^{k-1} n_{0}^{k-1}}{\pi^{2(k-1)} \varepsilon_{0}^{k-1} \varepsilon_{\infty}^{k-1} E_{0}^{k-1}} \times \exp \left(\frac{i \omega \tau}{W^{(0)}}\right) \times \\
\times \sum_{n_{1}=1}^{\infty} \sum_{n_{2}=1}^{\infty} \ldots \sum_{n_{k}=1}^{\infty}\left(\frac{\sin ^{2}\left(\frac{\pi n_{1}}{2}\right) \cdot \sin ^{2}\left(\frac{\pi n_{2}}{2}\right) \cdot \ldots \cdot \sin ^{2}\left(\frac{\pi n_{k}}{2}\right)}{n_{2}^{2} \cdot \ldots \cdot n_{k}^{2}\left(\frac{1}{\tau_{n_{1}}}+i \frac{\omega}{W^{(0)}}\right)\left(\frac{1}{\tau_{n_{2}}}+i \frac{\omega}{W^{(0)}}\right) \cdot \ldots \cdot\left(\frac{1}{\tau_{n_{k}}}+i \frac{\omega}{W^{(0)}}\right)}\right) \cos \left(\frac{\pi n_{1} a}{d} \xi\right) .
\end{gathered}
$$


From (1.1), when $r=1$, when

$$
\rho^{(\omega)}(\xi ; \tau)=\sum_{k=1}^{\infty} \gamma^{\mathrm{k}} \rho_{k}^{(\omega)}(\xi ; \tau),
$$

taking into account (13), we obtain (11).

\section{Non-linear polarization effects}

Polarization at the fundamental frequency $P^{(\omega)}(\xi, \tau)=q x \cdot \rho^{(\omega)}(\xi, \tau)$, averaged over the thickness of the dielectric

$$
P^{(\omega)}(\tau)=\frac{q}{d} \int_{0}^{d} x \rho^{(\omega)}(\xi, \tau) d x
$$

according to (11) it takes the form

$$
P^{(\omega)}(\tau)=\frac{8 a q n_{0} \gamma}{\pi^{2}\left(1-\frac{8 a q n_{0} \Lambda_{0} \gamma}{\pi^{2} \varepsilon_{0} \varepsilon_{\infty} E_{0}}\right)} \times \sum_{n=1}^{+\infty}\left[\frac{\sin ^{2}\left(\frac{\pi \mathrm{n}}{2}\right)}{n^{2}\left(\frac{1}{\tau_{n}}+i \frac{\omega}{W^{(0)}}\right)}\right] \times \exp \left(\frac{i \omega \tau}{W^{(0)}}\right) .
$$

Averaging $k$-th polarization components

taking into account (11) it gives

$$
P_{k}^{(\omega)}(\tau)=\frac{q}{d} \int_{0}^{d} x \rho_{k}^{(\omega)}(\xi, \tau) d x,
$$

$$
P_{k}^{(\omega)}(\tau)=\frac{8^{k} a^{k} q^{k} n_{0}^{k}}{\pi^{2 k} \varepsilon_{0}^{k-1} \varepsilon_{\infty}^{k-1} E_{0}^{k-1}} \sum_{n_{1}=1}^{\infty} \ldots \sum_{n_{k}=1}^{\infty}\left(\frac{\sin ^{2}\left(\frac{\pi n_{1}}{2}\right) \cdot \ldots \cdot \sin ^{2}\left(\frac{\pi n_{k}}{2}\right) \times \exp \left(\frac{i \omega}{a_{0}} \tau\right)}{n_{1}^{2} \cdot n_{2}^{2} \cdot \ldots \cdot n_{k}^{2} \cdot\left(\frac{1}{\tau_{n_{1}}}+i \frac{\omega}{W^{(0)}}\right) \cdot \ldots \cdot\left(\frac{1}{\tau_{n_{k}}}+i \frac{\omega}{W^{(0)}}\right)}\right) .
$$

Substituting (14) into (15)

$$
P^{(\omega)}(\tau)=\sum_{k=1}^{\infty} \gamma^{k} P_{k}^{(\omega)}(\tau)
$$

taking (18) confirms the expression (16).

As seen from the functions (13), (18), at the first odd field frequency $(\omega)$ manifest the nonlinear polarization phenomena is associated with the interaction relaxation modes of the same order perturbation theory $(k=1)$, differing in number $n_{k}$. The relaxation times for these modes $\tau_{n_{1}}, \tau_{n_{2}}, \ldots, \tau_{n_{k}}$. Obviously, a small parameter $\gamma$ of linear solution of the Fokker - Planck [2,3] is a special case of solutions of the generalized primary field frequency $(\omega)$ (11). Thus, a generalized formula for the polarization of the dielectric (16) in the first-order perturbation theory $(k=1)$ when $\sum_{\mathrm{k}=1}^{\infty}\left(\frac{8 \varphi n_{0} \Lambda_{0} \gamma}{\pi^{2}}\right)^{k-1}=\frac{1}{1-\frac{8 \varphi n_{0} \Lambda_{0} \gamma}{\pi^{2}}} \rightarrow 1$, proceeds to the result [2].

\section{Conclusions}

1. The influence of the nonlinearities of the kinetic equation of proton relaxation for mechanism of volumetric - charge polarization in proton semiconductors and dielectrics is investigated. From the solution of the nonlinear system of the equations of Fokker - Planck and Poisson (3), (4), recurrence formula for the complex amplitudes $\mathfrak{R}_{k}(n, \tau)$ of the relaxation mode number $\mathrm{n}$ in the k-th order perturbation theory is built (7).

2. By methods of quasiclassical kinetic theory it is proved that the forming space - charge polarization in the class HBC crystals in an alternating electric field only odd powers of the field relaxation harmonic $\rho^{((2 \lambda+1) \omega)}(\xi ; \tau) \sim E^{2 \lambda+1}(\mathrm{t})$ are generated in an external circuit. The generalized expression (16) is retrieved 
on the fundamental frequency of the alternating field $(\omega)$ for the polarization HBC with blocking contacts at the boundaries of the dielectric.

3. It is found that already at the first odd field frequency $(\omega)$ nonlinear effects caused by the interaction of different number $n_{k}$ relaxation modes $k=1$ order perturbation theory (equation (13), (18).

\title{
References
}

1 Медведев В.Я. Влияние туннелирования на диэлектрическую релаксацию / В.Я. Медведев, М.П. Тонконогов // Известия вузов. Физика. - 1987. - № 2. - С. 36-41.

2 Медведев В.Я. Туннельная миграционная поляризация в диэлектриках / В.Я. Медведев, М.П. Тонконогов // Известия вузов. Физика. - 1990. - № 11. - С. 71-75.

3 Калытка В.А. Протонная проводимость: монография. [Электронный ресурс] / В.А. Калытка, М.В. Коровкин. - Германия: Издательский дом LAP LAMBERT Academic Publishing, 2015. - 180 с. — Режим доступа: http://www.lappublishing.com.

4 Калытка В.А. Дисперсионные соотношения для протонной релаксации в твердых диэлектриках / В.А. Калытка, М.В. Коровкин // Известия вузов. Физика. — 2016. — Т. 59. — № 12. — С. 150-159.

\section{В.А. Калытка, М.В. Коровкин, Г.А. Вершинин, А.В. Баширов \\ Қатты диэлектриктердегі сызықтық емес көлемдік-зарядтық поляризация механизмі}

\begin{abstract}
Сызықты емес кинетикалық құбылыстар қалыптастыру кезінде көлемдік-зарядтық поляризация кристалдана отырып, сутектік кристалдық байланыстары бар (ӘКК) әдістерімен кинетикалық теория зерттелді. Бейсызық теңдеулер жүйесінің шешімін Фоккер-Планк және Пуассон блоктау электродтары деп аталатын математикалық сипаттау релаксация поляризация әлсіз өрістер (100-1000 кВ/м) және жоғары температура $(T>350)$ жеткілікті сызықтық жуықтау теориясы бойынша анықталып, тұжырымдалды. $n$ релаксациялық мода нөмірі бойынша аналитикалық түрде анықталған, яғни негізгі жиілікте айнымалы өріс байқалған, сызықтық емес әсерлер негізделген өзара іс-қимылмен ерекшеленді. Диффузия және қему коэффициенттері есептелуін ескере отырып, екі тетіктегі өтулер (термиялық белсенді және туннелді) арқылы параболалық протондар тосқауыл болады. Протонпротондық және протон-фонондық өзара әрекеттесу қаралмайды. Кинетикалық теңдеу шешімінің басқа да ұқсас ӘКК бойынша типі және қасиеттері кристалдық тордың, иондық өткізу кристалды байланыстар үшін ұсынылуы мүмкін.
\end{abstract}

Кілm сөздер: протонды жартылай өткізгіштер және диэлектриктер, сутектік байланыстары бар кристалдар, протондық релаксация және өткізгіштік, Фоккер-Планк және Пуассон сызықты емес теңдеулер жүйесі, релаксация модалары, поляризациялық жиілікті гармоникалар.

\section{В.А. Калытка, М.В. Коровкин, Г.А. Вершинин, А.В. Баширов \\ Механизм нелинейной объемно-зарядовой поляризации в твердых диэлектриках}

\begin{abstract}
Методами кинетической теории исследуются нелинейные кинетические явления при формировании объемно-зарядовой поляризации в кристаллах с водородными связями (КВС). Из решения нелинейной системы уравнений Фоккера - Планка и Пуассона при блокирующих электродах установлено, что при математическом описании релаксационной поляризации в КВС в области слабых полей $(100-1000$ кВ/м) и высоких температур $(T>350$ К) можно ограничиться первым приближением теории возмущений. Аналитически установлено, что на основной частоте переменного поля начинают проявляться нелинейные эффекты, обусловленные взаимодействием отличающихся по номеру $n$ релаксационных мод. Коэффициенты диффузии и подвижности вычисляются с учетом обоих механизмов переходов (термически активируемых и туннельных) протонов через параболический потенциальный барьер. Протон-протонное и протон-фононное взаимодействия не рассматриваются. Предложенная схема решения кинетического уравнения может быть применена и к другим, схожим с КВС по типу и свойствам кристаллической решетки, кристаллам с ионной приводимостью.
\end{abstract}


Ключевые слова: протонные полупроводники и диэлектрики; кристаллы с водородными связями (КВС); протонная релаксация и проводимость; нелинейная система уравнений Фоккера-Планка и Пуассона; релаксационные моды; поляризационные частотные гармоники.

\section{References}

1 Medvedev, V.Ya., \& Tonkonohov, M.P. (1987). Vliianie tunnelirovaniia na dielektricheskuiu relaksatsiiu [The influence of tunneling during the dielectric relaxation]. Izvestiia vysshikh uchebnykh zavedeniy. Fizika - Proceedings of Higher Educational Institutions. Physics, 2, 36-41 [in Russian].

2 Medvedev, V.Ya., \& Tonkonohov, M.P. (1990). Tunnelinaia mihratsionnaia poliarizatsiia v dielektrikakh [Tunneling migratory polarization in dielectrics]. Izvestiia vysshikh uchebnykh zavedeniy. Fizika - Proceedings of Higher Educational Institutions. Physics, 11, 71-75 [in Russian].

3 Kalytka, V.A., \& Korovkin, M.V. (2015). Protonnaia provodimost [Proton conductivity]. Germany: Izdatelskii dom «LAP LAMBERT Academic Publishing». lap-publishing.com. Retrieved from http://www.lap-publishing.com [in Russian]..

4 Kalytka, V.A., \& Korovkin, M.V. (2016). Dispersionnye sootnosheniia dlia protonnoi relaksatsii v tverdykh dielektrikakh [Dispersion relations for proton relaxation in solid dielectrics]. Izvestiia vuzov. Fizika - Proceedings of high schools. Physics, 59, $12,2151-2161$. 